\title{
Human "memories" can be evoked by stimulation of the lateral temporal cortex after ipsilateral medial temporal lobe resection
}

\author{
J L Moriarity, D Boatman, G L Krauss, P B Storm, F A Lenz
}

\begin{abstract}
A patient with medically intractable seizures and mesial temporal sclerosis underwent a left anterior temporal lobectomy and amygdalohippocampectomy. After 4 months, his seizures recurred and a left temporal, subdural grid of electrodes was placed to localise his seizure focus. Stimulation through the grid evoked four distinct "memories", or experiential phenomena, despite absence of the ipsilateral medial temporal lobe. To our knowledge, this is the first documented case of experiential phenomena evoked by cortical stimulation in the absence of the ipsilateral medial temporal lobe.

(F Neurol Neurosurg Psychiatry 2001;71:549-551)
\end{abstract}

Keywords: experiential phenomena; hippocampectomy; cortical stimulation; memory

The localisation of memory within the human brain is a fundamental question in neuroscience. Since the end of the 19th century, there has been debate about the relative importance of the medial temporal structures, ${ }^{12}$ and the lateral temporal cortex, ${ }^{3}$ or both, in the production of memories evoked by stimulation of the brain (experiential phenomena ${ }^{4}$ ). We now address this issue directly through the study of a patient in whom familiar songs and voices were evoked by electrical stimulation of the lateral temporal cortex after removal of ipsilateral medial temporal lobe stuctures.

Department of Neurosurgery, Meyer Building 7-113, Johns Hopkins Hospital, 600 North Wolfe Street, Baltimore, $M D$ 21287-7713, USA J L Moriarity P B Storm F A Lenz

Department of Neurology

D Boatman

G L Krauss

Correspondence to:

F A Lenz

fal@pallidum.med.jhu.edu

Received 19 October 2000 and in revised form

2 May 2001

Accepted 16 May 2001 function. A left anterior temporal lobectomy and amygdalohippocampectomy was carried out.

The postoperative MRI demonstrated that the resection of the hippocampus and parahippocampal gyrus extended to the level of the tectal plate (figure $1 \mathrm{~A}$ ). Parts of the amygdala above the initial segment of the middle cerebral artery and parts of the fusiform gyrus were not removed. Postoperative neuropsychological testing (see methods) showed no significant change from the preoperative situation except that tests of short term auditory memory (digit span) showed a mild decrease in performance.

Four months after the surgery the patient began having daily simple and complex partial seizures with left temporal lobe onset. Fourteen months after his initial surgery, a subdural grid of monitoring electrodes was implanted over the left lateral temporal cortex to localise his seizures. Cortical stimulation through the grid was carried out to map language function. During this stimulation, the patient reported four distinct experiential phenomena (see below). Monitoring showed seizure onsets over the anterior/inferior aspect of the grid. This focus was resected 7 days after implantation of the grid. At that time the completeness of the hippocampectomy was confirmed by direct visualisation.

\section{Methods}

Before the first surgery, the patient underwent standardised neuropsychological testing. He scored within normal limits on all tests of short term memory and new verbal learning (digit span, Wechsler memory test, Rey auditory verbal learning test), and on tests of long term auditory memory (Rey auditory verbal learning test). The patient had a verbal IQ of 92 (30th percentile), performance IQ of 104 (60th percentile), and a full scale IQ of 96 (40th percentile)(Wechsler adult intelligence scalerevised).

On the day before the amobarbital test the patient underwent a pretest on a practice set to familiarise him with the protocol and establish baseline performance levels. During the test intracarotid amobarbital (125 $\mathrm{mg}$ in $1.5 \mathrm{ml}$ warmed saline) was hand injected into the internal carotid artery (left side first) after a routine angiogram disclosed no vascular abnormalities. The patient was monitored for changes in 
counting, limb strength, and the EEG. Speech and language testing began after the patient demonstrated no voluntary movement of the contralateral arm and marked slowing of the ipsilateral EEG. Language testing was performed in the following order for both sides: object naming, auditory comprehension, speech discrimination, single word reading, picture naming, and picture-word relatedness.

The injection of amobarbital included 37 $\mathrm{MBq}$ of radiotracer technetium $99 \mathrm{~m}-$ hexamethylpropyleneamine oxime for single photon emission tomography (SPECT) to evaluate the qualitative distribution of amobarbital during testing. The SPECT study was performed within 1 hour of the injections and confirmed tracer distribution throughout the medial temporal lobes bilaterally.

During the second surgery a grid of platinum iridium cortical electrodes (2.3 $\mathrm{mm}$ diameter) were implanted over the left cerebral hemisphere as depicted in figure $1 \mathrm{~B}$. Electrode position was determined by intraoperative observation and verified by postoperative three
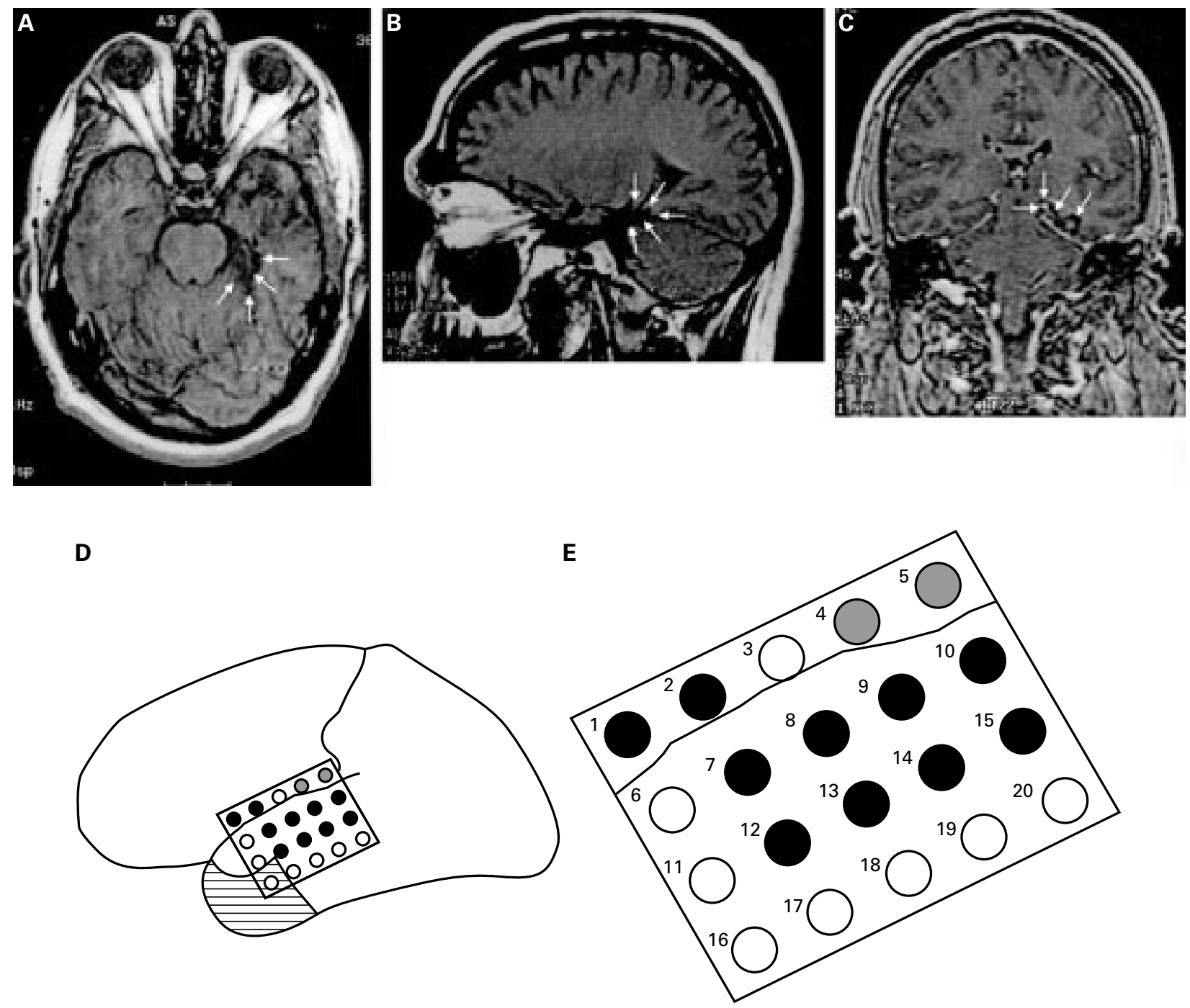

Response to stimulation

Specific experiential response

None

Focal motor activity

1-2 Flintstones cartoon

9-10 Flintstones cartoon contralateral side of tongue

10-15 Flintstones cartoon

Experiential phenomenon

13-14 Pink Floyd

8-9 Pink Floyd

9-10 Baseball announcer

7-8 Female voice singing

12-13 Female voice singing

Figure 1 (A) Axial, (B) sagittal, and (C) coronal MRI views of the patient's brain. Arrows depict the extent of the left medial temporal lobe resection. (D) Position of grid over left temporal lobe and its relation to the sylvian fissure and central sulcus estimated from radiography, CT, MRI, and cortical stimulation studies plus intraoperative observation. Region marked with horizontal lines represents the extent of anterior and lateral temporal lobectomy.

(E) Detail of left temporal grid. Intercontact distance along the rectangular grid lines is $1 \mathrm{~cm}$. 
dimensional CT. Cortical stimulation was performed over 2 days to localise motor and language functions. Square wave pulses, of 0.3 $\mathrm{ms}$ duration and alternating polarity, were generated in 5 second trains ( 50 pulses/s) between pairs of adjacent electrodes. Testing was performed at the maximal current threshold (10-15 mA) that did not produce afterdischarges, defined as rhythmic EEG activity sustained beyond termination of the stimulation. Testing was only performed when at least five stimulated trials could be obtained without afterdischarges. The mean number of repetitions at any site was 15 . EEG recordings were obtained with Grass Model 12 amplifiers (bandpass: $0.1-100 \mathrm{~Hz}$, amplifier gain 5000). Electrode impedance was maintained at less than $20 \mathrm{kohm}$.

\section{Results}

On the first day of cortical stimulation the patient's aura was evoked, consisting of the theme song from the Flintstones cartoon, which he had watched from 5-15 years of age. The patient reported hearing "a lot of the Flintstones" at three electrode pairs (9-10, 10-15, and 1-2) tested on the first day. Because numerous afterdischarges were elicited by stimulation of these three electrode pairs, additional testing was not performed at these sites on that day.

The three other experiential phenomena were repeatedly evoked, in the absence of afterdischarges, on the second day of stimulation testing when the patient's antiepileptic drug concentrations were higher than on the first day. The second experiential phenomenon occurred with stimulation at electrodes $13-14$ and 8-9. As stimulation proceeded he was able, during stimulation, to repeat the words that he was hearing and to identify the song as "Wish you were here" by the musical group Pink Floyd. When questioned later, the patient reported listening to that song from the age of 11 to the present. The third experiential phenomenon, produced by stimulation of electrode pair 9-10 was the voice of a baseball announcer (Richie Ashburn) calling an unidentified game involving the Philadelphia Phillies. The patient reported listening to this announcer throughout his childhood and adult life until the announcer's death in 1997. The fourth phenomenon occurred with stimulation at electrodes $7-8$ as well as $12-13$. The patient consistently reported hearing a familiar female voice singing, although he could not identify the singer or the song.

\section{Discussion}

Neuropsychological testing of amnestic patients $^{5}$ as well as lesioning studies in nonhuman primates have firmly established that formation of declarative memory (of facts, events) depends on the integrity of the hippocampus and the cortex overlying the amygdala. ${ }^{6}$ However, amnestic patients with medial temporal lobe damage can recall remote events as well as normal subjects but cannot recall recent events. ${ }^{57}$ Thus the role of the medial temporal lobe in memory is thought to be transient so that at some point, recall of a given memory becomes hippocampus independent and perhaps cortically dependent, ${ }^{8}$ consistent with studies in animals. ${ }^{9}$ The present report suggests that this model may also apply to human stimulation evoked experiential phenomena, as it demonstrates that they can be evoked by stimulation of superior lateral temporal neocortex in the absence of ipsilateral medial temporal lobe structures.

For two reasons, it seems unlikely that spread of neural activation to the right hippocampus accounts for our findings. Firstly, anatomical evidence demonstrates much stronger communication between the neocortex and its ipsilateral than contralateral hippocampus. ${ }^{10}$ Secondly, preoperative amobarbital testing demonstrated that inactivation of the distribution of the left internal carotid including medial temporal lobe (SPECT) suppressed auditory verbal memory. However, if there were not some residual memory function in the right hippocampus the patient would have been profoundly amnestic after the first surgery. ${ }^{5}$ Finally the result in this epileptic patient might not apply to the normal population because memory may have shifted from the medial temporal lobe as a result of the disease. ${ }^{11}$

Over a century ago, John Hughlings Jackson described memories or experiential phenomena occurring during temporal lobe seizures and attributed these to discharges arising in the medial temporal lobe or uncinate region. ${ }^{1}$ Over the next century these phemomena were attributed to the cortex of the superior temporal gyrus, ${ }^{3}$ to the medial temporal lobe, ${ }^{2}$ or to a combination of medial and lateral temporal structures. ${ }^{4}$ The present case is the first report of experiential phenomena evoked by cortical stimulation in the absence of ipsilateral medial temporal structures. It demonstrates that these structures are not required for experiential phenomena evoked by stimulation of the superior lateral temporal cortex.

This work was supported by grants to FAL from the NIH (P01This work was supported by grants to FAL from the NIH (P01Rowland and D Johnson for technical assistance.

1 Hughlings Jackson J. On right or left sided spasm at the onset of epileptic paroxysms, and on crude sensation warnings, and elaborate mental states. Brain 1880;3:192-206.

2 Gloor P, Olivier A, Quesney LF, et al. The role of the limbic system in experiential phenomena of temporal lobe epilepsy. Ann Neurol 1982;12:129-44.

3 Fiendel W, Penfield W. Localization of discharge in temporal lobe automatism. Archives of Neurology and Psychiatry 1954;72:605-30.

4 Bancaud J, Brunet-Bourgin F, Chauvel P, et al. Anatomical origin of déjà vu and vivid "memories" in human temporal lobe epilepsy. Brain 1994;117:71-90.

5 Penfield W, Milner B. Memory deficits induced by bilateral lesions in the hippocampal zone. Archives of Neurology and lesions in the hippocampal
Psychiatry 1958;79:475-97.

Psychiatry 1958;79:475-97.
Milner B, Squire LR, Kandel E. Cognitive neuroscience and Milner B, Squire LR, Kandel E. Cognitive neurose
the study of memory. Neuron 1989;20:445-68

7 Rempel-Clower NL, Zola SM, Squire LR, et al. Three cases of enduring memory impairment after bilateral damage limited to the hippocampal formation. I Neurosci 1996;16: 5233-55.

8 Markowitsch HJ. Which brain regions are critically involved in the retrieval of old episodic memory? Brain Res Brain Res Rev 1995;21:111-27.

9 Zola-Morgan S, Squire LR. The primate hippocampal formation: evidence for a time-limited role in memory storage. Science 1990;250:288-90.

10 Tranel D, Brady DR, Van Hoesen GW, et al. Parahippocampal projections to posterior auditory association cortex (area Trojections to posterior auditory association cortex (area

11 Henke K, Wieser HG. Bilateral medial temporal lobe damage without amnesic syndrome: a case report. Epilepsy Res 1996;24:147-61. 